\title{
FPGA Implementation of AHB to APB Protocol
}

\author{
Sowmya Aithal ${ }^{1}$, Dr. J. S. Baligar ${ }^{2}$, Guruprasad S. P. ${ }^{3}$ \\ ${ }^{\mathbf{1}}$ PG Student, Dr. Ambedkar Institute of Technology, Bangalore, India \\ ${ }^{2}$ Associate Professor, Dept. of ECE, Dr. Ambedkar Institute of Technology, Bangalore, India \\ ${ }^{3}$ Senior Design Engineer, Certitude TechnologiesPrivate Limited, Bangalore, India
}

\begin{abstract}
The field of technology is evolving at a very fast pace. The competition is very intense. So the need of the hour is to produce efficient system. In accomplishing this objective we are required to establish better interaction among all the components of the system. This requirement is fulfilled by the Advanced Microcontroller Bus Architecture (AMBA) protocol from Advanced RISC Machines (ARM).The AMBA is the on-chip standard for the communication among components in Application Specific Integrated Circuits (ASIC) or System on Chip (SoC). This paper focuses on the 2 protocols of AMBA which are Advanced High Performance Bus (AHB) and Advanced Peripheral Bus (APB) and theAPB bridge. The coding is done in Verilog synthesis on Xilinx 14.7 ISE and simulation on ISim simulator and FPGA implementation on Spartan 3.
\end{abstract}

Keywords: AMBA; APB bridge, AHB, APB, IP, SoC, Verilog ,VLSI

\section{Introduction}

The miniaturization in the technology is leading to the emphasizing on the communication among the modules of the System on Chip (SoC). The SoC is the integration of all the components required onto the same chip so called System on Chip. The interaction between these components of the system is critical for every SoC. The intercommunication is maintained by AMBA protocol. The AMBA protocol provides an efficient way for the interaction and increases the performance of the system.

In figure 1 the components like Direct Memory Access (DMA), Random Access Memory (RAM), External Memory Interface, ARM processor are the components in SoC and the peripheral components like Universal Asynchronous Receiver Transmitter (UART), Timer, Keypad, Programmable Input Output (PIO). The communication here is established by AHB on the master side and by APB for the peripheral side. The bridge provides the interconnection between AHB to APB.

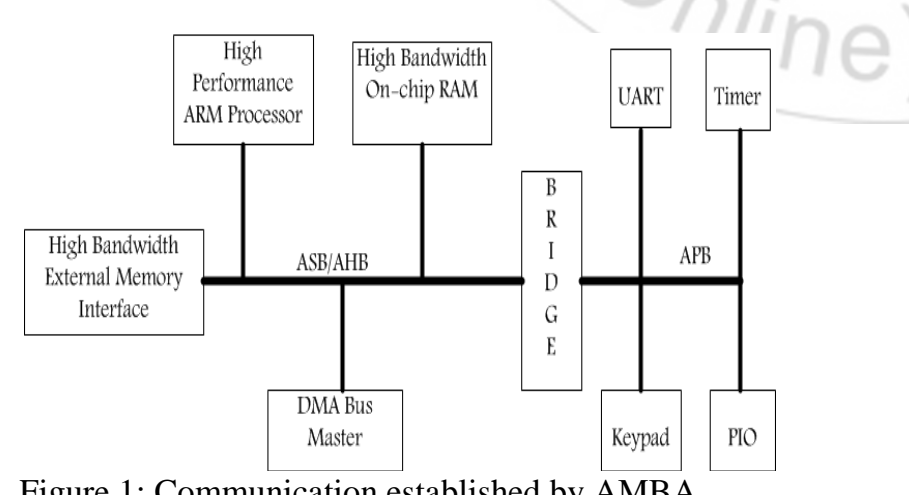

Figure 1: Communication established by AMBA

\section{Related Work}

The paper [1] describes the design of AHB to APB module. It describes the AHB module and the designing of AHB to APB Bridge. The coding is done in Verilog. Here the AHB monitor or driver is designed. The paperexplains only on the RTL simulation and is not implemented on FPGA.
The paper [2] describes about the APB protocol its comparison with AHB and ASB. The paper explains the state diagram of $\mathrm{APB}$ and describes the signals required for the APB and the coding is done in Verilog. The paper explains only about APB protocol and is good for understanding APB protocol.

The paper [3] describes about the AHB module and is coded in VHDL. The AHB signals are learnt and design of AHB arbiter is understood. The AHB module can be understood for implementation with the aid of this paper.

The paper [4] describes how an efficient Finite State Machine (FSM) for the AHB master with the understanding of the various signals of AHB master can be designed. The operation of $\mathrm{AHB}$ master is required for the designing an efficient FSM and thus the AHB master. The AHB module can only be designed.

The paper [5] describes the design of AHB arbiter with the emphasis on AHB arbiter architecture. The arbiter logic is explained. The paper basically is for design of arbiter for AHB in Verilog.

The paper [6] describes the design of incrementing burst transfer for AHB high performance. In this paper the focus is on the burst transfers and its capability of extracting high performance from AHB. The paper uses Verilog for coding AHB burst performance.

The paper [7] compares between the AMBA bus protocols of its version 2.0 that are AHB, ASB and APB. The comparison is based on their application to performance. The paper highlights the different buses and the features of the buses.

The paper [8] describes the design of AHB to APB module for different frequencies and phase. The paper explains the design of AHB to APB and their performance for different frequencies. The paper explains the design and helps in understanding of the interface between two protocols.

The paper [9] describes the performance comparison between various versions of AMBA protocols. 


\section{International Journal of Science and Research (IJSR) \\ ISSN (Online): 2319-7064 \\ Index Copernicus Value (2013): 6.14 | Impact Factor (2015): 6.391}

he paper [10] describes the methodology for AHB master wrappers for its use in Intellectual Property (IP) cores.

\section{AHB Bus Protocol}

The AHB is the high performance bus and synthesizable designs.It supports burst transfers, split transactions, nontristate implementation. The burst transfers is the block of data which is sent at a fast pace. The block of data can vary from 8 to 16 beat bursts. The split transfers referred to option for splitting the beat bursts into smaller beats and transferring. The non tristate implementation is not including high impedance state. Every transfer consists of an address and control cycle, one or more cycles for the data. The AHB bus master has signals for writing, data buses, address buses, burst transfers, etc. The AHB bus master when ready for the transfer will check if peripheral device is ready for the transfer if ready then the transaction takes place. The reverse operation occurs when read transfer happens. The AHB controller controls the transaction between AHB master and APB peripheral.

\section{APB Bus Protocol}

The APB protocol is the bus protocol employed for the peripherals like UART, Keypad, PIO, Timer, LCD Display or LED display etc to name a few.

The APB protocol is low performance low power bus protocol. It is basically used for peripherals as the name suggests. The APB protocol has low bandwidth.

The APB peripherals as listed can be enabled based on their enable signals and the transaction can be accomplished.

The APB peripherals in this work is enabled based on the address and so is fast and efficient.
Table 1: Device Utilization Summary for Spartan 3.

\begin{tabular}{|c|c|c|c|}
\hline Logic Utilization & Used & Available & Utilization \\
\hline Number Of Slices & 90 & 3584 & $2 \%$ \\
\hline Number of Slice FlipFlops & 154 & 7168 & $2 \%$ \\
\hline Number of 4input LUT's & 117 & 7168 & $1 \%$ \\
\hline Number of IOBs & 282 & 141 & $200 \%$ \\
\hline Number of GCLK's & 3 & 8 & $37 \%$ \\
\hline
\end{tabular}

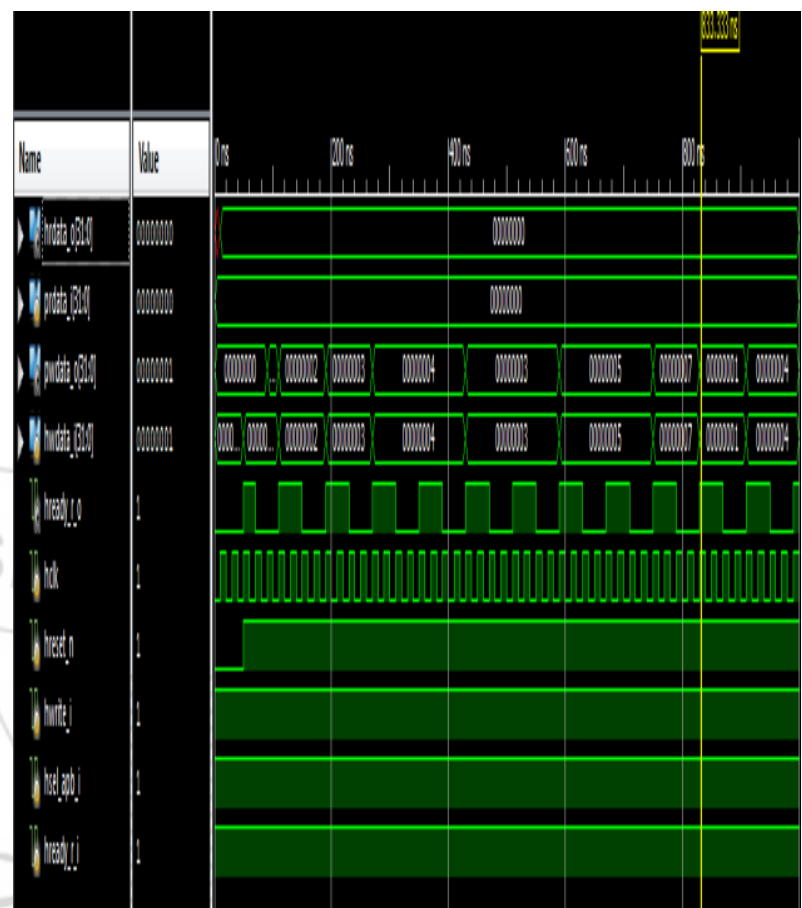

Figure 2: Simulation Result of AHB to APB

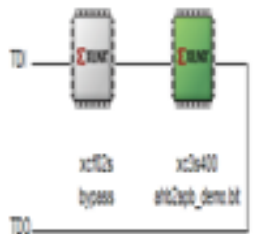

\section{AHB to APB Module}

The AHB to APB module consists of the AHB signals and APB signals included to form the controller part and the peripherals which include 6 of the slaves.

The AHB to APB module is coded in Verilog and synthesized in Xilinx 14.7 ISE and simulated using ISIM simulator which is inbuilt in Xilinx 14.7.

The AHB to APB module can be implemented on Spartan 3 FPGA and usingChipscope can be verified. The higher Spartan series also is compatible and the AHB to APB module can be dumped onto it.

\section{Results}

Figure 3: Dumping on to the FPGA

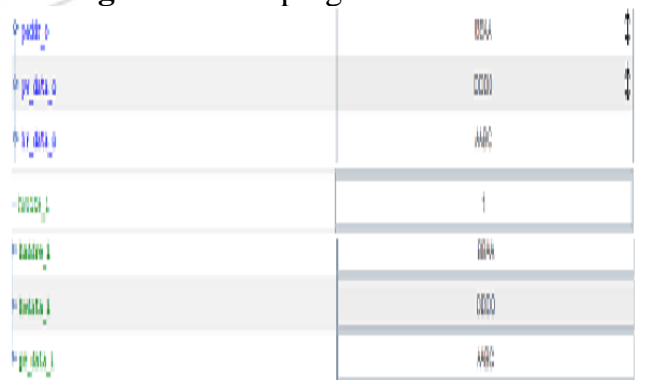

Figure 4: Chipscope results for Write Operation.

The synthesis results are shown along with the simulation results.

1) Minimum period: $5.018 \mathrm{~ns}$

2) Maximum Frequency: $199.299 \mathrm{MHz}$

3) Minimum input arrival time before clock: 6.429ns

4) Maximum output required time after clock: $14.120 \mathrm{~ns}$

5) Maximum combinational path delay: $8.525 \mathrm{~ns}$ 


\section{International Journal of Science and Research (IJSR) \\ ISSN (Online): 2319-7064}

Index Copernicus Value (2013): 6.14 | Impact Factor (2015): 6.391

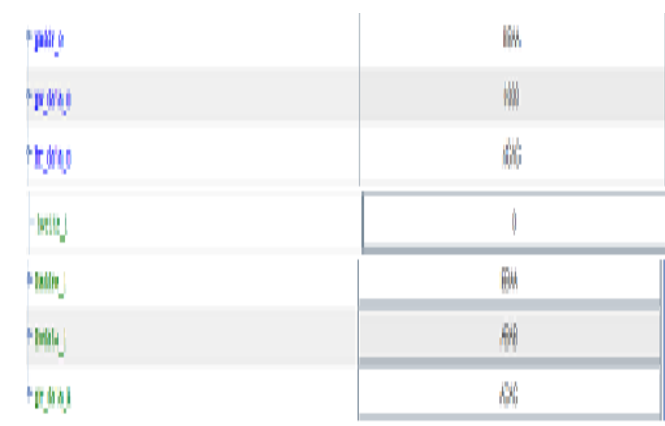

Figure 4: Chipscope results for Read Operation.

The figures 2, 3, 4, 5 show the simulation results, with remaining figures illustrating the dumping onto FPGA and the Chipscope results or both write and read operations.

The table 1 shows the device utilization for Spartan 3 FPGA and it shows the utilization of resources to the minimal. The Chipscope results verify the design.

\section{Conclusion and Future Work}

The AHB to APB module is synthesized, simulated and verified by dumping onto the Spartan 3 FPGA. The Chipscope results for both write and read operations are verified. The area, time consumed by the design is low and so we can conclude as a very effective design.

The AHB to APB module can be dumped onto any other Spartan Series Kits also for similar verification

The design of the interface between $\mathrm{AHB}$ and $\mathrm{APB}$ can be extended into other versions of the AMBA protocol like ASB, AXI to name a few.

The implementation of interface between other protocols of AMBA with APB is the future work.

\section{References}

[1] Design of AMBA Based AHB2APB Bridge by Vani.R.M andM.Roopain IJCSNS International Journal of Computer Science and Network Security, 2010.

[2] Design \& Implementation of Advance Peripheral Bus Protocol by Heli Shah, ChinmayModi, BhargavTarpara in International Journal of Scientific Engineering and Applied Science (IJSEAS),2015.

[3] Implementation of AMBA AHB protocol for high capacity memory management using VHDL by Varshavishwarkama,Abhishekchoubey,ArvindSahu in International Journal on Computer Science and Engineering (IJCSE), 2012.

[4] Design of an Efficient FSM for an Implementation of AMBA AHB Master by Pravin S. Shete,Dr. ShrutiOza, in International Journal of Advanced Research in Computer Science and Software Engineering, 2014.

[5] Study Of High Performance AmbaAhb Reconfigurable Arbiter For On-Chip Bus Architecture by Pravin S. Shete,Dr. ShrutiOza, International Journal of Electrical, Electronics and Data Communication, 2014.
[6] Design Incrementing Burst Data Transfer Operation for AMBA-Advanced High Performance Bus by MitalMungra and Assi. Prof. Vishal S.Vora in International Journal of Emerging Trends in Electrical and Electronics (IJETEE), 2013.

[7] An Overview of Advanced Microcontroller Bus Architecture relate on APB bridge by RadhikaKoti, DivyaMeshram in International Journal of Scientific and Research Publications, 2013.

[8] Design of AHB2APB Bridge for different phase and Frequency by Vani.R.M andM.Roopa in International Journal of Computer and Electrical Engineering, 2011.

[9] Performance Comparison of AMBA Bus-Based SystemOn-Chip Communication Protocol by AnuragShrivastava G.S. Tomar, Ashutosh Kumar Singh in International Conference on Communication Systems and Network Technologies, 2011.

[10] A methodology for the design of AHB bus master wrappers by Marc Bertola, Guy Bois in IEEE ,2003.

[11] AMBA Specifications from ARM. 